\title{
AVALIAÇÃO DO MARCO REGULATÓRIO NA PRODUÇÃO DE MEDICAMENTOS FITOTERÁPICOS NO BRASIL
}

\author{
Sonia Cristina Dantas de Brito \\ Universidade Federal de Tocantins (UFT) \\ Waldecy Rodrigues \\ Universidade Federal de Tocantins (UFT)
}

\begin{abstract}
AVALIAÇÃO DO MARCO REGULATÓRIO NA PRODUÇÃO DE MEDICAMENTOS FITOTERÁPICOS NO BRASIL
Resumo: Para analisar a legislação para fitoterápicos no Brasil e as mudanças propostas com o Projeto de Lei $n$. $^{\circ}$ 3381/2004, que tenta modernizá-la, foi utilizado o Delphi Eletrônico, que busca o consenso de opiniões entre especialistas na área. Assim, foram aplicados 100 questionários e, a partir dos resultados, foi possível constatar que a atual legislação é uma das maiores barreiras do setor, devido às exigências e altos custos com pesquisas, assim como a falta de estudo sobre as plantas nativas, o que privilegia a entrada de plantas exóticas no país. A falta de informação, descrédito e desconfiança dos profissionais prescritores, também, têm prejudicado o setor. A pesquisa conclui, portanto, que se o Projeto de Lei for aprovado, trará mudanças positivas como: influência no comportamento das indústrias brasileiras, aumentando sua produção; alteração na localização geográfica das indústrias deste setor, causando a descentralização; aumento na utilização de plantas nativas que, consequentemente, ocasionará a diminuição nos níveis de importação.

Palavras-chave: Plantas Medicinais, fitoterápicos, legislação.
\end{abstract}

\section{EVALUATION OF THE REGULATORY MARK IN THE PRODUCTION OF PHYTOTHERAPEUTIC MEDICINES IN BRAZIL}

Abstract: To analyze the legislation of phytotherapeutic in Brazil and the changes proposed by the Law Project 3381/2004, that tempts to modernize it, was utilized the Electronic Delphi, that looks for opinion consensus among specialists of the area. Therefore, one hundred questionnaires were applied and the results discovered that the current legislation is one of the biggest limits to the production chain, due to the exigencies and high costs with researches, as well as the lack of study about native herbs, that makes easier the entrance of exotic plants in the country. The lack of information, discredit and suspicion of the prescriber professionals, also, have prejudiced of the area. Concludes that if the Law Project comes to be approved, it will bring positive changes like: influence in the behavior of Brazilian industries, increasing its production; change of geographical place of the industries of this sector, causing decentralization; improve the use of native herbs that, consequently will cause a decrease in the importation levels.

Key words: Medicinal plants, phytotherapeutics, legislation. 


\section{INTRODUÇÃO}

Nas últimas décadas vem sendo observada uma crescente consciência sobre os bens e serviços oferecidos pela natureza e a sua importância para garantir o bem-estar dos indivíduos, de acordo com Pavarini (2000). Fonte de imenso potencial de uso econômico, os componentes da biodiversidade podem fornecer uma ampla gama de produtos de importância econômica. Dentre esses produtos destacam - se os fitoterápicos que são, conceitualmente, medicamentos preparados exclusivamente a base de plantas medicinais (GUERRA; NODARI, 2003).

SegundooMinistério da Saúde, ofitoterápicoéo medicamento obtido empregando-se exclusivamente matérias-primas ativas vegetais, caracterizado pelo conhecimento da eficácia e dos riscos de seu uso, assim como pela reprodutibilidade e constância de sua qualidade (BRASIL, 2004). As plantas, atualmente, são utilizadas para fins medicinais por países desenvolvidos e em desenvolvimento. Nos primeiros, como uma alternativa mais saudável, ou menos danosa, de tratamento, e como resultante do não acesso aos medicamentos farmoquímicos, no caso dos países em desenvolvimento (BRASIL, 2007).

No Brasil, a competitividade da cadeia produtiva de medicamentos fitoterápicos possui alguns pontos que podem ser considerados como os principais para impulsionar a indústria deste setor como: o desenvolvimento de tecnologia, suas condições de apropriabilidade e a escala de produção uma vez que tal cadeia estende-se desde a produção agrícola à produção industrial e sua comercialização (HASENCLEVER, 2009).

O Brasil sendo um país megadiverso, porque não desenvolve uma indústria competitiva de medicamentos fitoterápicos. São vários fatores intervenientes, dentre os quais se destacam: a produção altamente concentrada em grandes laboratórios multinacionais; o alto custo dos procedimentos de pesquisa que vão desde a bioprospecção até a fase dos testes de segurança (toxicologia pré-clínica e clínica) e de eficácia terapêutica (farmacologia pré-clínica e clínica) exigidos pela atual legislação; a ausência de uma política industrial e tecnológica mais efetiva para o estímulo de pesquisas e exploração econômica das plantas medicinais e a falta de uma legislação mais moderna que permita aos laboratórios nacionais investirem na produção de medicamentos a partir de plantas medicinais (RODRIGUES; NOGUEIRA, 2008).

No comércio exterior o Brasil apresenta-se como um importador líquido em todos os segmentos da cadeia produtiva de medicamentos fitoterápicos. Em torno de 1/3 das importações do país em 2008 proveram, principalmente, da Alemanha e países, além da China (HASENCLEVER, 2009).
Desde 2000, quando foi publicada a RDC 17/2000 - Agência Nacional de Vigilância Sanitária (ANVISA), atualmente revogada pela RDC 48/2004, que trouxe novos critérios para o registro dos medicamentos fitoterápicos, de acordo com as indústrias fitoterápicas nacionais vem vivendo uma situação de crise. As etapas exigidas para o registro de um medicamento fitoterápico, que vão da bioprospecção até a fase dos testes préclínicos e clínicos, tornaram elevados os custos com pesquisas, transformando-se em uma das barreiras mais fortes deste setor.

A importância desta pesquisa reside na necessidade de identificar a atual situação da cadeia produtiva de medicamentos fitoterápicos no Brasil e os efeitos nesta de possíveis alterações em seu marco regulatório, tornando-o mais flexível. Neste cenário, a eficácia clínica poderia ser respaldada na evidência científica ou em literatura que comprove seu uso etnocultural.

\section{MÉTODOS}

Para este estudo, utilizou-se o método Web Delphi ou Delphi Eletrônico. De acordo com Giovinazzo e Fischmann (2001), este método é uma ferramenta de pesquisa qualitativa que busca um consenso de opiniões de um grupo de especialistas a respeito de eventos futuros, conservando as mesmas premissas características de uma pesquisa Delphi tradicional. É mantido o anonimato dos respondentes, a representação estatística da distribuição dos resultados e o feedback de respostas do grupo para reavaliação nas rodadas seguintes, sendo que os resultados da primeira rodada são divulgados na Internet, para que possam ser considerados pelo grupo no preenchimento da segunda rodada (MARTINO, 1993).

Assim, a pesquisa passou por sete etapas, que configuram a metodologia do Delphi Eletrônico:

1. Definição do objetivo da pesquisa - neste caso, o objetivo está em apresentar a atual situação da cadeia produtiva de medicamentos fitoterápicos no Brasil e os efeitos sobre esta, a partir da introdução de um marco regulatório mais flexível e identificar outros gargalos competitivos.

2. Elaboração do questionário da 1﹎. rodada - para esta rodada foram elaboradas 15 questões do tipo: concorda ou discorda.

3. Seleção dos especialistas - foi realizada a partir de buscas na internet de atores envolvidos com o setor, obtendose um número de 100 especialistas divididos em diversas categorias: consultores, professores/pesquisadores (mestre e doutores), profissionais de 
saúde (nutricionistas, farmacêuticos e naturólogos), membros de associações e autônomos. Esses especialistas representavam instituições: acadêmicas, governamentais e não - governamentais, de todas as regiões do país.

4. Preenchimento do Questionário pela Internet - Os respondentes foram comunicados por e-mail sobre a disponibilidade do questionário que se deu através de um software de pesquisa online.

5. Aplicação de Estatística e Análise dos Resultados - Para a tabulação e análise dos dados da $1_{-}^{0}$ rodada, foi utilizada a estatística simples.

6. Elaboração do questionário da $2_{-}^{\circ}$ rodada - conforme metodologia, foi preparada apresentando-se os resultados da $1_{-}^{\circ}$ rodada, possibilitando aos respondentes a revisão de sua opinião, diante da posição e argumentação do grupo. O questionário, nesta rodada, também foi disponibilizado para preenchimento através de um software de pesquisa online. Com esta rodada, a pesquisa foi encerrada, alcançando-se, para a pesquisa, o consenso satisfatório entre os especialistas.

7. Conclusões gerais e relatório final-Com os resultados, produziu-se um artigo, o qual foi enviado por e-mail aos participantes.
Para Giovinazzo e Fischmann (2001), o Método Delphi Eletrônico possui algumas vantagens adicionais em relação ao Delphi tradicional, pois esta variação elimina: a utilização dos correios ou outros serviços de entrega para o envio dos questionários, o que reduz drasticamente os custos na preparação dos materiais e envio; o tempo necessário para a realização da pesquisa, também é reduzido; os questionários são respondidos diretamente em um formulário na Internet; permite um feedback muito mais rápido aos respondentes; traz a vantagem de utilizar uma mídia mais atraente e flexível, o que torna o preenchimento do questionário mais agradável e eficiente.

Participaram deste estudo especialistas de todas as regiões do país, sendo: $30 \%$ da Norte, $21 \%$ da Nordeste, $21 \%$ da Sudeste, $14 \%$ da CentroOeste e $14 \%$ da Sul. Foram especialistas de diversas categorias, sendo: $44 \%$ professores pesquisadores (mestres e doutores), 29\% consultores de instituições federais, $16 \%$ profissionais de saúde (nutricionistas, farmacêuticos e naturólogos), 7\% membros de associações e 4\% profissionais autônomos.

$\mathrm{Na}$ primeira rodada da pesquisa foram enviados 100 questionários, com uma abstenção de $55 \%$. Na segunda rodada foram enviados 45 questionários, com abstenção de $10 \%$. Na primeira rodada, a abstenção apresentou-se um pouco acima dos valores encontrados em literatura que, em geral, ficam entre $30 \%$ e $50 \%$. Já na segunda rodada, a abstenção ficou um pouco abaixo. Em geral, segundo Wright e Giovinazzo (2000), nesta rodada, os valores ficam entre $20 \%$ e $30 \%$.

\section{Gráfico 1 - Os atuais obstáculos da cadeia produtiva de medicamentos fitoterápicos, segundo os} especialistas

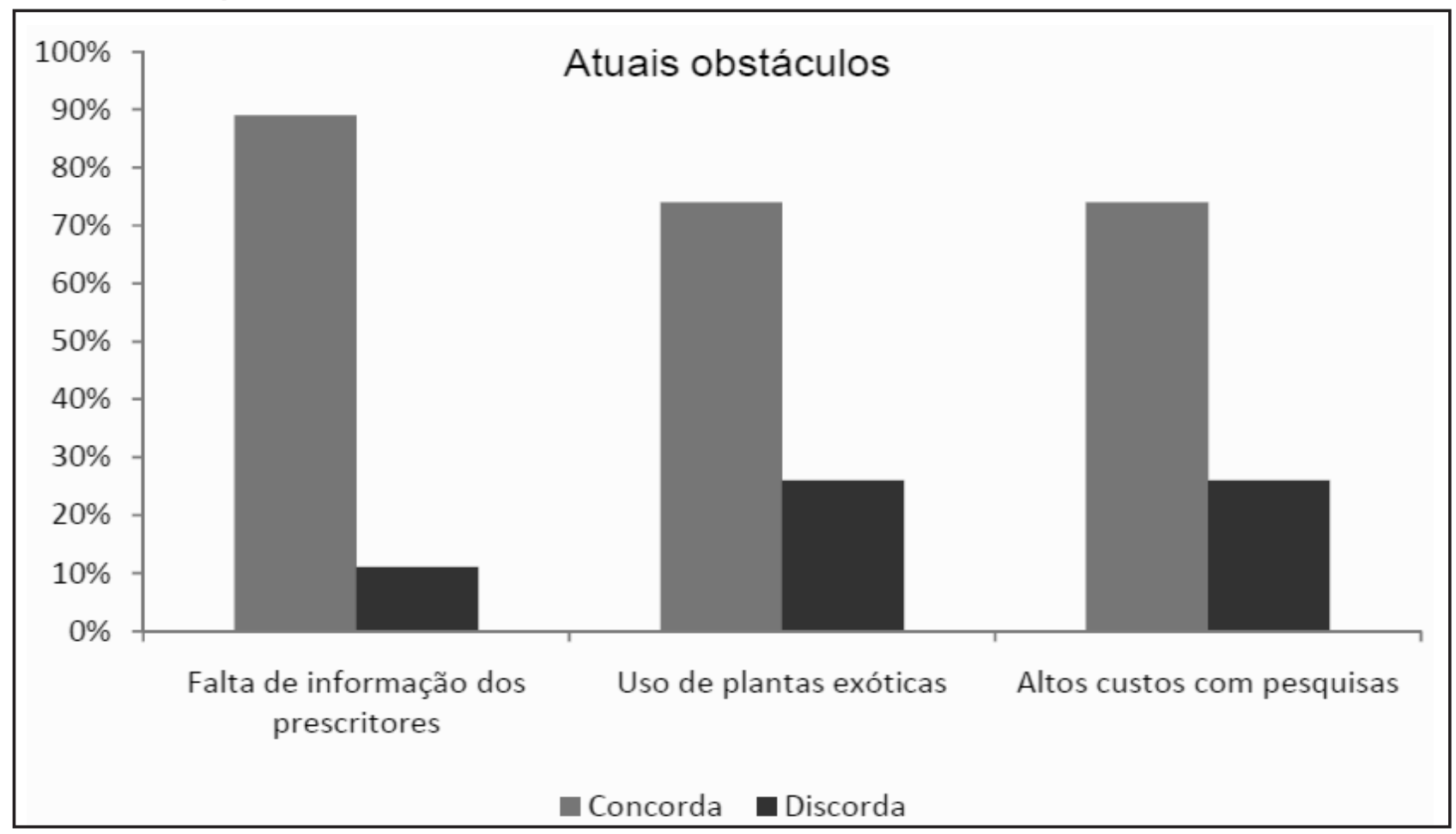

Fonte: Elaborado pelos autores. 


\section{RESULTADOS}

De acordo com os especialistas, existem vários obstáculos que prejudicam o desenvolvimento da cadeia produtiva de medicamentos fitoterápicos. Entre os principais apontados pelos peritos estão: a falta de informação para prescritores (89\%); o uso de plantas exóticas $(74 \%)$ e os altos custos com pesquisas $(74 \%)$, Gráfico 1 .

Os peritos afirmam que existe uma forte resistência dos prescritores em receitar medicamentos fitoterápicos. Porém, o parecer n.o 04/92 do Conselho Federal de Medicina, em 17 de janeiro de 1992, mostra que a fitoterapia foi reconhecida como um método terapêutico. No entanto, ainda se percebe certo preconceito e falta de informação por grande parte destes profissionais.

$\mathrm{Em}$ relação à legislação aplicada à produção de medicamentos fitoterápicos, para os participantes, os riscos com os altos custos com pesquisas são ampliados com o atual modelo de registro na ANVISA. Este modelo segue o padrão europeu, onde os requisitos são: a comprovação da eficácia terapêutica, a comprovação da qualidade, tanto da matéria-prima utilizada quanto do produto final, e estudos de toxicidade que definam o grau de risco do produto Ministério da Saúde (BRASIL, 2004). Bem diferente da legislação canadense que não exige a realização de testes clínicos, onde os mesmos devem ter a qualidade e segurança comprovada, porém a eficácia deve ser respaldada na evidência científica ou em literatura que comprove seu uso etnocultural. Assim, a legislação brasileira para o setor de fitoterápicos, por reforçar o critério de segurança e eficácia dos produtos registrados no Brasil, acaba privilegiando, de certa forma, a incorporação de plantas exóticas no país.

Porém, o governo vem introduzindo medicamentos fitoterápicos na atenção básica à saúde desde 2007, quando o Sistema Único de Saúde (SUS) passou a fornecer medicamentos à base de Espinheira - Santa (Maytenus Ilicifolia) e Guaco (Mikania glomerata). Atualmente, na Relação Nacional de Plantas Medicinais de interesse ao SUS, fazem parte 71 espécies de plantas medicinais.

Para os especialistas, as ações governamentais podem trazer grandes melhorias para a cadeia produtiva de medicamentos fitoterápicos. Cerca de $90 \%$ dos entrevistados consideram como fundamentais a implantação de programas agrícolas para manejo das plantas medicinais cultivadas, a inserção dos fitoterápicos no SUS e o aumento das compras governamentais. Contudo, destaca-se que essas ações encontramse previstas nas diretrizes da Política Nacional de Plantas Medicinais e Medicamentos Fitoterápicos (PNPMF), Gráfico 2.

Uma importante mudança institucional, para o setor de medicamentos fitoterápicos no Brasil, é esperada com a aprovação do Projeto de Lei (PL) n. ${ }^{\circ} 3381$, de 15 de abril de 2004. Tal lei prevê a modernização da legislação brasileira para obtenção

\section{Gráfico 2 - Ações governamentais para melhorias no setor de fitoterápicos}

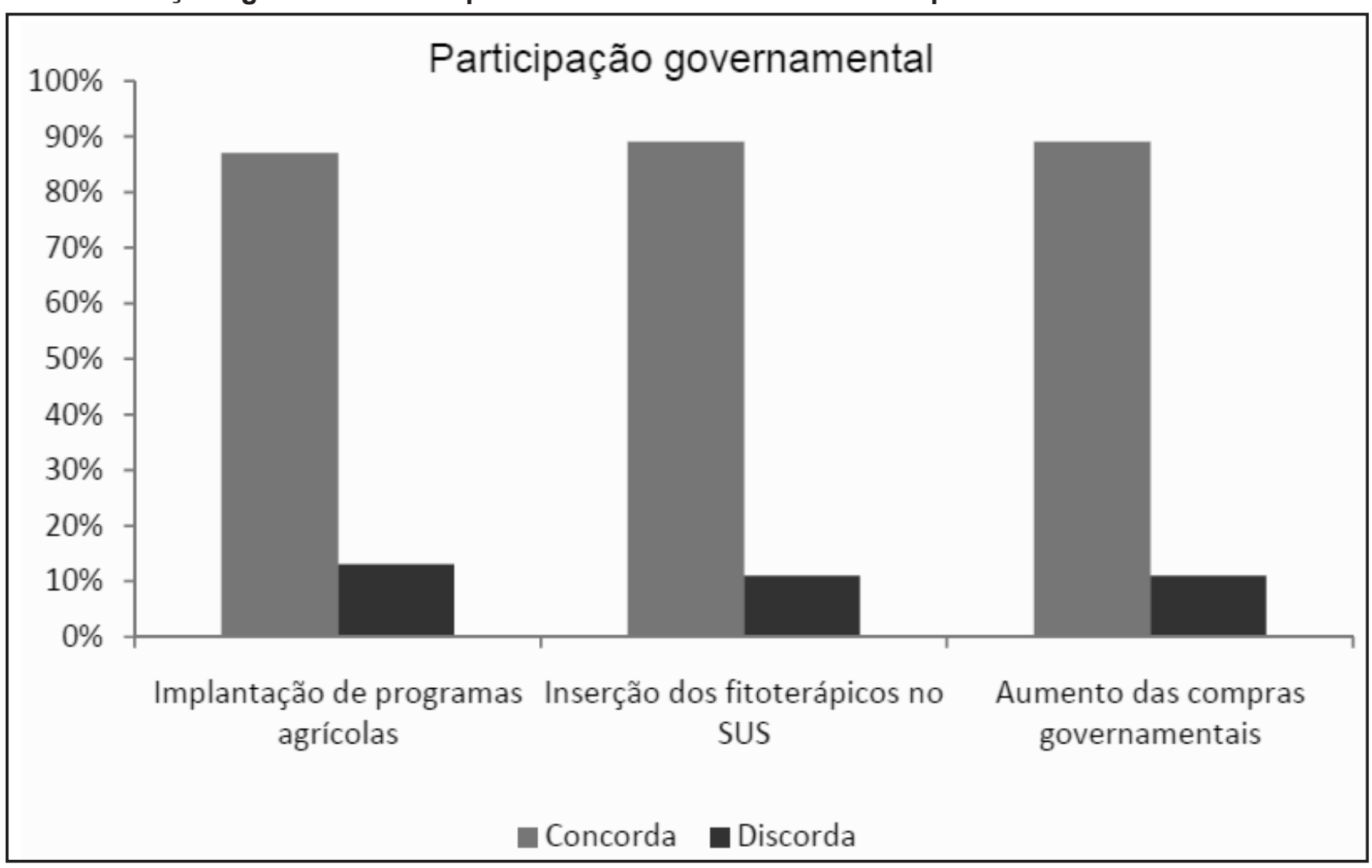

Fonte: Elaborado pelos autores. 
de registro de medicamentos fitoterápicos, inspirada na legislação canadense, que regulamenta os Natural Health Products (produtos de origem natural para a saúde). No Brasil, o PL criaria uma nova categoria denominada Produtos para Promoção da Saúde (PPS), que registrará a maior parte dos medicamentos fitoterápicos brasileiros. Assim, a legislação proposta retira a necessidade da realização de testes clínicos destes produtos, que devem ter a qualidade e a segurança comprovadas, porém a eficácia deve ser respaldada na evidência científica ou em literatura que comprove seu uso etnocultural (RODRIGUES; NOGUEIRA, 2008).

Dessa forma, caso o PL seja aprovado, de acordo com a previsão dos especialistas, algumas mudanças na cadeia produtiva irão acontecer. Para $68 \%$ dos respondentes, essas mudanças, que ocorrerão, serão totalmente favoráveis ao mercado de medicamentos fitoterápicos no país. Para $82 \%$ destes, o Projeto de Lei influenciará, de forma positiva, no comportamento das indústrias brasileiras que passarão a produzir mais e, para $78 \%$, mais plantas medicinais pertencentes ao bioma brasileiro serão consumidas; cerca de $71 \%$ acreditam na ampliação da dispersão geográfica das indústrias de medicamentos fitoterápicos no país e $60 \%$ veem uma diminuição no nível de importação de plantas medicinais e de seus extratos, Gráfico 3.

\section{DISCUSSÃO}

Sob o ponto de vista dos especialistas do setor, percebeu-se que a atual legislação para o registro de medicamentos fitoterápicos originou obstáculos para o setor, devido às exigências para tal atividade; um deles está nos altos custos com pesquisas que passam por etapas que vão da bioprospecção até a fase dos testes pré-clínicos e clínicos.

Por esse motivo, as indústrias brasileiras perderam o interesse na produção deste tipo de medicamento, especialmente, as indústrias de pequeno porte. Atualmente, as pesquisas neste setor são realizadas mais frequentemente por universidades, porém tais estudos não seguem as normas exigidas pelas leis para o registro, tornando as pesquisas inutilizáveis para fins comerciais (SIMÕES; SCHENKEL, 2002).

De acordo com Ribeiro, Leite e Dantas-Barros (2005), as universidades brasileiras já demonstram que têm competência para formar recursos humanos capacitados e gerar conhecimentos, mas não têm o dinamismo necessário para transformar os resultados de seus trabalhos em produtos finais de aplicação tecnológica. Para Simões e Schenkel (2002), a aproximação da indústria farmacêutica com a academia tornou-se a base: da sua sobrevivência, da geração de novos empregos, e da formação de

Gráfico 3 - As mudanças na cadeia produtiva de medicamentos fitoterápicos, no caso da aprovação do Projeto de Lei $n .^{\circ} 3381 / 2004$, para os especialistas

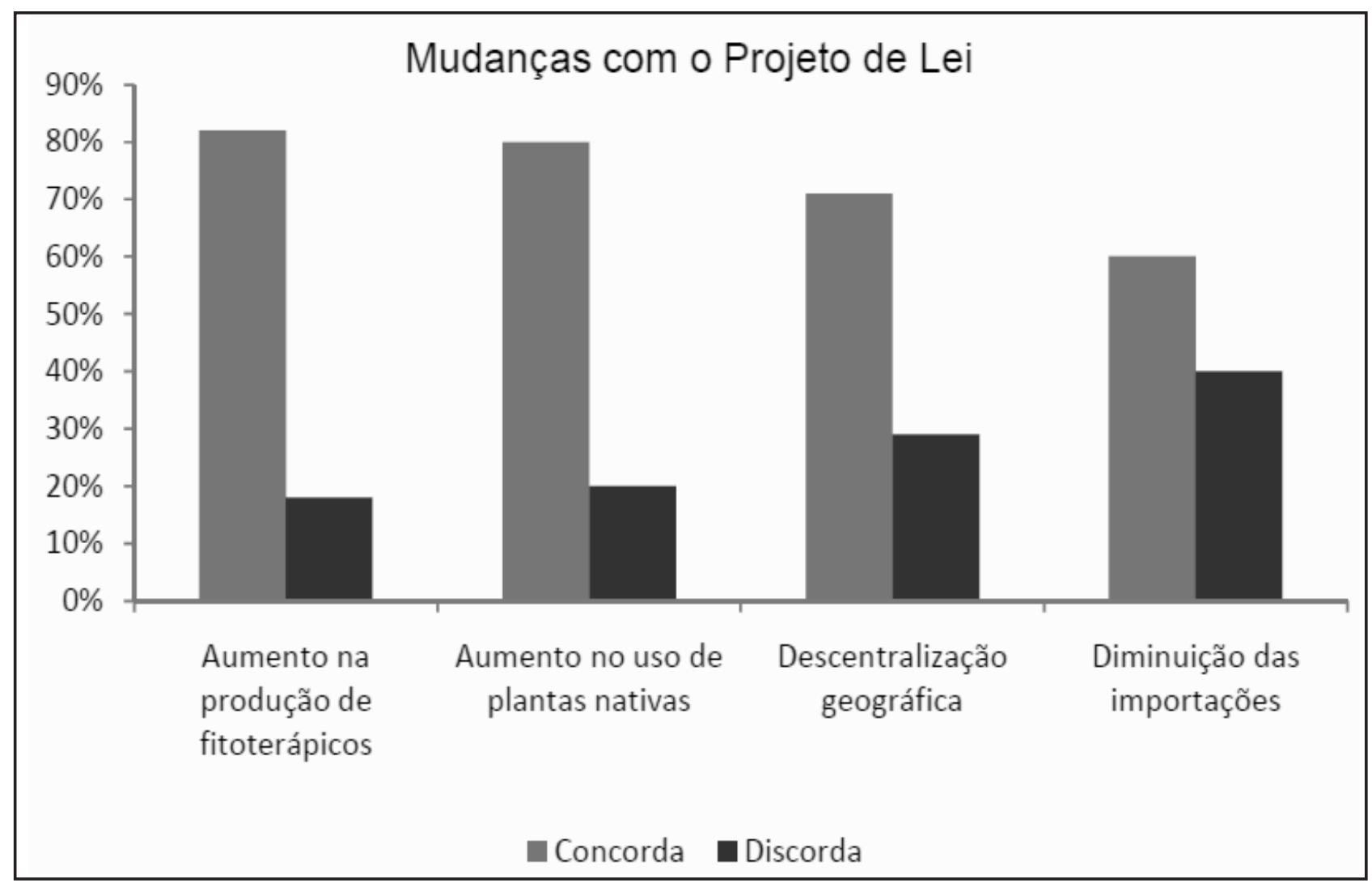

Fonte: Elaborado pelos autores. 
uma nova mentalidade, que estabelece o uso dos recursos naturais propiciando o desenvolvimento socioeconômico, com o indispensável respeito aos ecossistemas e buscando a manutenção das características culturais do país.

Além dos altos custos com as pesquisas o setor tem problemas, também, com a falta de profissionais qualificados na realização deste tipo de trabalho. Em consequência disso, surge outra barreira nesta cadeia produtiva, a utilização de plantas exóticas na produção de medicamentos fitoterápicos, pois, atualmente, a maioria das obras na literatura, colocadas como referência, não contemplam estudos clínicos de plantas da flora brasileira utilizadas na medicina popular (RIBEIRO; LEITE; DANTAS-BARROS, 2005).

Portanto, percebe-se que a atual legislação, com suas exigências para o registro de medicamentos fitoterápicos, acarreta grandes barreiras para o setor, porém, de acordo com os especialistas, o maior obstáculo não acontece em consequência destas ações. Este vem da falta de credibilidade e informação por parte de grande maioria dos profissionais prescritores, em relação a este tipo de produto. Além disso, de acordo com os entrevistados, existem possíveis parcerias entre tais profissionais e laboratórios de medicamentos sintéticos, o que acabam por influenciar no momento da prescrição. Contudo, este descrédito e desconfiança não provêm apenas dos médicos, mas, também, de parte dos pacientes, e esta questão pode ser resolvida com a divulgação de informações aos profissionais prescritores, a criação de mais cursos de especialização em fitoterapia e a introdução de uma disciplina, no curso de medicina, voltada para este tema.

Em 2006, foram aprovadas duas políticas importantes para o setor: a Política Nacional de Práticas Integrativas e Complementares (PNPIC) no SUS e a PNPMF. De acordo com Carvalho e outros (2008), ambas apresentam em suas diretrizes o incentivo à pesquisa e desenvolvimento deste setor, priorizando a biodiversidade do país, além de estimularem a adoção da Fitoterapia nos programas de saúde pública. Assim, a partir da criação destas políticas e a introdução dos fitoterápicos no SUS, houve um aumento na utilização destes medicamentos, porém, muitas das plantas utilizadas como base na produção ainda são exóticas.

Essa inclusão de fitoterápicos, na atenção básica à saúde, é uma medida que já vem sendo tomada desde 2007, quando o SUS passou a fornecer produto à base de espinheira - santa (Maytenus Ilicifolia), utilizada para tratar gastrites e úlceras e guaco (Mikania glomerata) utilizado no tratamento de tosses e gripes, em diversas apresentações. O objetivo do Ministério da Saúde é ampliar, futuramente, a lista destes medicamentos. Segundo os especialistas, esta medida traz uma maior credibilidade a este tipo de medicamento, além de que aumentaria a sua demanda e estimularia a cultura dos fitoterápicos no país.

$\mathrm{Na}$ Relação Nacional de Plantas Medicinais de interesse ao SUS estão incluídas, atualmente, 71 espécies de plantas medicinais, porém, dentre estas, $47 \%$ são plantas exóticas, em geral, nativas da Europa, Ásia e África.

A Política Pública PNPMF, aprovada em 2006, tem como uma de suas diretrizes promover um programa de inclusão da agricultura familiar nas cadeias e nos arranjos produtivos das plantas medicinais, insumos e fitoterápicos (BRASIL, 2007). Esse incentivo agrícola tornaria o país independente de matéria-prima importada, ou seja, diminuiria a dependência externa, o que geraria enormes benefícios saídos do campo até os laboratórios, trazendo um efeito imediato no custo de produção dos medicamentos fitoterápicos, aumentando a produção de medicamentos locais; além disso, esta ação levaria à inserção de novas plantas medicinais nos inventários fitoterápicos do país.

Portanto, sendo o Brasil um país com grande biodiversidade, este número de plantas exóticas poderia ser muito menor, se mais estudos de plantas nativas fossem realizados. Segundo Barbosa (2010), no cerrado brasileiro, por exemplo, apesar da sua riqueza florística e de seu uso medicinal pela população, apenas dezessete espécies, dentre 270 com potencial medicinal, foram identificadas como fonte de princípio ativo para medicamentos fitoterápicos pela ANVISA. Isto demonstra a intensa necessidade de realização de estudos das plantas nativas.

Sobre o PL n. ${ }^{\circ} 3381 / 2004$, que tem como objetivo modernizar a legislação brasileira para o registro de medicamentos fitoterápicos, a partir da criação de uma nova categoria denominada PPS, observou-se que, com a aprovação, de acordo com os especialistas, ocorrerão grandes melhorias nesta cadeia produtiva.

Os entrevistados acreditam que os custos com pesquisas que, atualmente, são vistos como uns dos principais gargalos seriam menores, pois, esta nova categoria de medicamentos por ser mais flexível, não exigirá as etapas mais onerosas (testes pré-clínicos e clínicos) neste tipo de pesquisa. Sendo assim, a eficácia clínica poderá ser respaldada na evidência científica ou em literatura que comprove seu uso etnocultural. Portanto, com esta diminuição de custos, se comparada aos medicamentos sintéticos, os fitoterápicos passariam a ter prioridade na produção. Para os especialistas, esse aumento será em torno de $25 \%$.

$\mathrm{Na}$ visão dos peritos, outro aumento que ocorrerá, será na quantidade de plantas nativas utilizadas na fabricação de fitoterápicos, pois com a comprovação da eficácia, baseada em evidência científica e uso etnocultural, um maior número de plantas do bioma brasileiro passariam a ser utilizadas na fabricação de novos medicamentos 
fitoterápicos. Atualmente, poucas plantas nativas estão incluídas na Farmacopéia Brasileira. Portanto, com esse aumento, mais plantas medicinais nativas passariam a fazer parte da lista.

A aprovação do PL facilitará a descentralização geográfica que, atualmente, concentra-se na Região Sudeste no eixo Rio de Janeiro - São Paulo. Isso poderá influenciar na qualidade das plantas utilizadas na produção dos medicamentos fitoterápicos, através da produção com clima, temperatura e solo adequados.

Por fim, com o aumento na utilização de plantas medicinais nativas, consequentemente, o número de importações de plantas medicinais e extratos diminuirá, pois haverá mais estudos com plantas nativas. Assim, essa diminuição, na previsão dos especialistas, será em torno de 10\%.

\section{CONCLUSÃO}

Logo, percebe-se que as alterações na atual legislação para o registro de medicamentos fitoterápicos trarão mudanças positivas para esta cadeia produtiva, como o acesso fácil da população a este tipo de terapia. Porém, não se pode esquecer da qualidade dos produtos que serão oferecidos e nem dos benefícios que devem ser proporcionados à saúde brasileira através destes.

Com a Política Nacional de Medicamentos Fitoterápicos, muitos dos problemas desta cadeia produtiva, apresentados neste estudo, poderão ser resolvidos promovendo melhorias no acesso da população a este tipo de medicamento e, além de valorizar o conhecimento tradicional das comunidades, basta apenas que esta seja colocada em prática, completamente.

\section{REFERÊNCIAS}

BARBOSA, G. F. Plantas Medicinais: alternativa econômica a conservação do cerrado brasileiro? 2010. 95 f. Dissertação (Mestrado) - Programa de Pós-Graduação Mestrado em Desenvolvimento Regional e Agronegócio, Universidade Federal do Tocantins, Tocantins, 2010.

BRASIL. Ministério da Saúde. Agência Nacional de Vigilância Sanitária. Resolução de Diretoria Colegiado $n^{\circ}$. 48, de 16 de março de 2004. Aprova o regulamento técnico de medicamentos fitoterápicos junto ao Sistema Nacional de Vigilância Sanitária. Diário Oficial da União, Brasília, DF, 2004.

Estrutura de mercado do segmento de fitoterápicos no contexto atual da indústria farmacêutica brasileira. Brasília, DF, 2007. Área de Economia as Saúde e Desenvolvimento.
CARVALHO, A.C.B. et. al. In: Situação do registro de medicamentos fitoterápicos no Brasil. Revista Brasileira de Farmacognosia, Curitiba, v. 18, n. 2, p. 314-319, 2008.

GIOVINAZZO, R. A.; FISCHMANN, A. A. Delphi Eletrônico: uma experiência de utilização da metodologia da pesquisa e seu potencial de abrangência regional. In: CONGRESSO LATINOAMERICANO DE ESTRATÉGIA, 14., 2001, Buenos Aires. Anais ... Buenos Aires: SLADE, 2001.

GUERRA, P. M.; NODARI, R. O Biodiversidade: aspectos biológicos geográficos, legais e éticos. In: SIMÕES, Cláudia Maria Oliveira et al. (Orgs.). Farmacognosia da planta ao medicamento. 5. ed. rev. e ampl. Porto Alegre: Editora da UFRHS; Florianópolis: Editora da UFSC, 2003.

HASENCLEVER, L. Diagnóstico dos desafios e oportunidades no mercado de Fitoterápicos Brasileiro - Análise da Balança Comercial Brasileira e Depósitos de Patentes no Brasil. Centro de Gestão e Estudos Estratégicos - CGEE. Rio de Janeiro, 2009.

MARTINO, J. P. Technological forecasting for decision making. 3. ed. New York: Mc Graw-Hill Inc., 1993.

PAVARINI, M. de F. Prospecção da diversidade biológica: perspectiva para o caso brasileiro. 2000. 88 f. Tese (Doutorado) - Programa de PósGraduação de Engenharia, Universidade Federal do Rio de Janeiro, Rio de Janeiro, 2000. Disponível em:<http://www.ppe.ufrj.br/ppe/production/tesis/ mpavarini.pdf>. Acesso em: 29 jan. 2011.

RIBEIRO, A. Q.; LEITE, J. P. V.; DANTAS-BARROS, A. M. Perfil de utilização de fitoterápicos em farmácias comunitárias de Belo Horizonte sob a influência da legislação nacional. Revista Brasileira de Farmacognosia, Curitiba, v. 15, n. 1, p. 65-70, 2005.

RODRIGUES, W.; NOGUEIRA, J. M. Competitividade da Cadeia Produtiva de Plantas Medicinais no Brasil: uma perspectiva a partir do comércio exterior. Informe Gepec, Toledo, v. 12, n. 2, p. 91-105, 2008.

SIMÕES, C. M. O., SCHENKEL, E. P. A Pesquisa e a produção brasileira de medicamentos a partir de plantas medicinais: a necessária interação da indústria com a academia. Revista Brasileira de Farmacognosia, Curitiba, v. 12, n. 1, p. 35-40, 2002.

WRIGHT, J. T. C.; GIOVINAZZO, R. A. Delphi: uma ferramenta de apoio ao planejamento prospectivo. Caderno de Pesquisas em Administração, São Paulo, v. 1, n. 12, p. 54-65, 2000. 


\section{Sonia Cristina Dantas de Brito}

Engenheira Ambiental

Mestre em Desenvolvimento Regional e Agronegócio pela Fundação Universidade Federal do Tocantins (UFT)

Pesquisadora do Núcleo de Desenvolvimento Regional da Universidade Federal de Tocantins (UFT)

E-mail: eng.soniabrito@yahoo.com.br

\section{Waldecy Rodrigues}

Economista

Doutor em Ciências Sociais pela Universidade de Brasília (UnB)

Professor Adjunto do Mestrado em Desenvolvimento Regional e Pró-Reitor de Pesquisa e Pós-Graduação da Universidade Federal de Tocantins (UFT)

E-mail: waldecy@terra.com.br

\section{Universidade Federal de Tocantins - UFT}

Av NS 15, 109 Norte, Plano Diretor Norte - Palmas - TO CEP: 77001-090 